Revista Economía y Administración, Vol. 9, No. 22018

\title{
INTERDEPENDENCIA Y COMPLEMENTARIEDAD ENTRE PYME'S COMO FACTOR DE LA PRODUCTIVIDAD Y COMPETITIVIDAD
}

(1) Doris Noelia Zuniga Alvarado

Universidad Nacional Autónoma de Honduras (UNAH)

doris.zunigaalvarado@gmail.com

Doraydee Castellon Vogel

Universidad Nacional Autónoma de Honduras (UNAH)

doraydee.castello@unah.edu.hn

DOI: $\underline{\text { http://dx.doi.org/10.5377/eya.v9i2.6658 }}$

\section{RESUMEN}

La productividad y competitividad de las empresas son factores de gran relevancia para el éxito de las mismas, pero esto depende en gran medida de la Interdependencia y Complementariedad que pueda existir entre ellas. Cabe mencionar, que las pequeñas y medianas empresas (Pymes no son la excepción). Es por tal razón que en el presente artículo se abordaran cuatro importantes variables Interdependencia, Complementariedad, Productividad y Competitividad.

Cuando se habla del tema de competitividad es hablar de varias fases o ejes, un importante escritor que habla de la competitividad y la ha definido claramente es Michael Porter, para él "la ventaja competitiva de un país "es su capacidad para incitar a las empresas (locales o extranjeras) a utilizar el país como plataforma para llevar adelante sus actividades. Para determinar las fuerzas y las debilidades competitivas de los países y sus principales sectores" (Centro de Comercio Internacional, 2013). Estrategia y metodología seguir La investigación tiene un enfoque cuantitativo Tipo y Alcance de Investigación Correlacional ya que se pretende relacionar variables y contrastar los resultados para ver cuál es la relación que existe entre las variables. Con un diseño de la Investigación No Experimental Transversal

En cuanto al aporte de Aporte la investigación es constructivista porque pretende aportar conocimiento útil para la Academia, las Empresas y el País se usará un método dialectico hermenéutico para la comprensión, la explicación y la interpretación de las variables planteadas.

Palabras Clave: Pymes, Interdependencia, Complementariedad, Productividad y Competitividad.

(1) Autor de contacto 


\section{INTERDEPENDENCE AND COMPLEMENTARITY BETWEEN SMES AS A FACTOR OF PRODUCTIVITY AND COMPETITIVENESS}

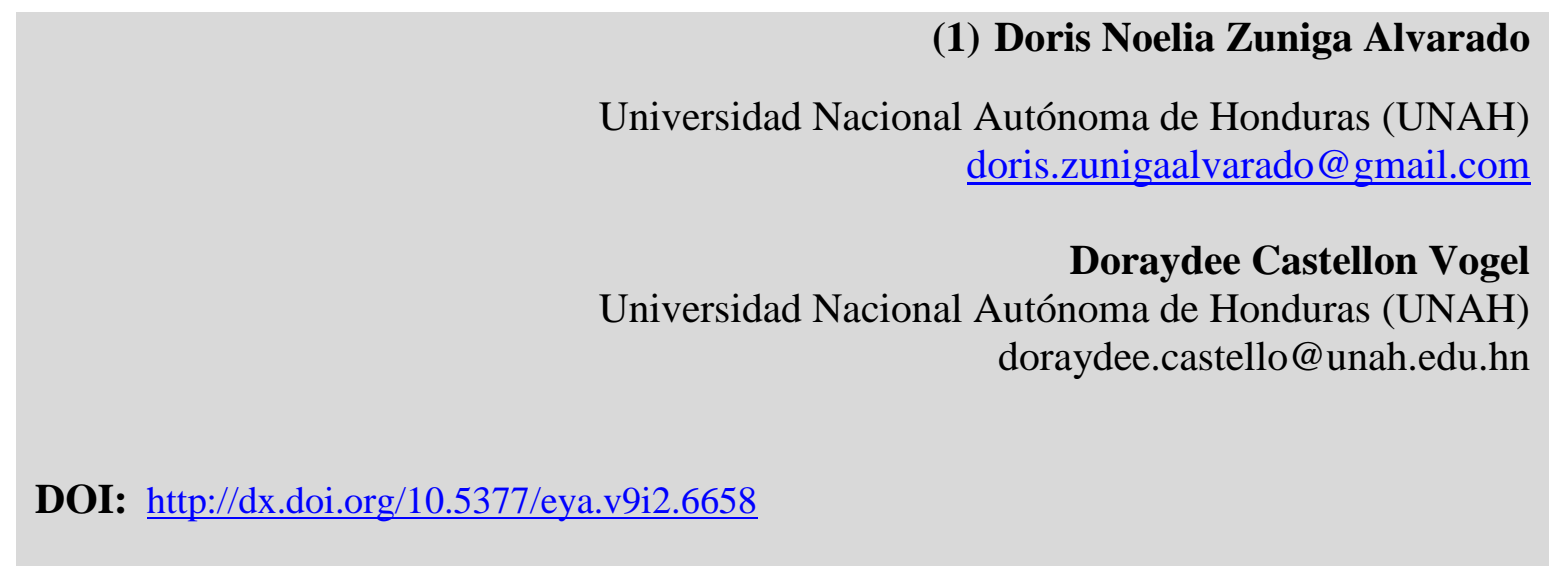

\section{ABSTRACT}

The productivity and competitiveness of companies are factors of great relevance for their success, but this depends largely on the interdependence and complementarity that may exist between them. It is worth mentioning that small and medium enterprises (SMEs are not the exception). For this reason, this article will address four important variables Interdependence, Complementarity, Productivity and Competitiveness.

When talking about the issue of competitiveness is talking about several phases or axes, an important writer who talks about competitiveness and clearly defined is Michael Porter, for him "the competitive advantage of a country" is its ability to encourage companies (local or foreign) to use the country as a platform to carry out their activities. To determine the competitive strengths and weaknesses of countries and their main sectors "(International Trade Center, 2013).

Strategy and methodology to follow the research has a quantitative approach Type and Scope of Correlational Research since is intended to relate variables and compare the results to see what is the relationship between the variables? With a design of the Transversal Non-Experimental Investigation. Regarding the contribution, the research is constructivist because it intends to provide useful knowledge for the Academy, the Companies and the Country, a dialectic hermeneutical method for understanding, explaining and interpreting the variables proposed.

Keywords: SMEs, Interdependence, Complementarity, Productivity and Competitiveness 


\section{INTERDEPENDENCIA Y COMPLEMENTARIEDAD ENTRE PYME 'S COMO}

FACTOR DE LA PRODUCTIVIDAD Y COMPETITIVIDAD

Revista Economía y Administración, Vol. 9, No. 22018

"Panificadoras" para poder profundizar

(1) Corresponding author

\section{INTRODUCCIÓN}

Con el objetivo de una mayor comprensión se hace un análisis acerca de la Interdependencia y Complementariedad y como esta incide en la productividad y competitividad de las mismas, si se evalúa la situación de las empresas hondureñas se encontrará que no existe una gran diferencia en la situación que presentan las pymes a nivel de Latinoamérica y la situación problemática que presentan en Honduras, razón por la cual el presente artículo puede ser de gran utilidad.

\section{Explicación del objeto de estudio y posición epistemológica del escritor}

El presente estudio tiene como objetivo principal determinar cuál es la Interdependencia y Complementariedad que existe en la actualidad en las empresas Pymes y como aportan estas a la Productividad y Competitividad de las mismas, así como la importancia de las mismas en el país, tomando como caso de estudio un sector industrial aún más en la investigación.

$\mathrm{Si} \quad$ la Interdependencia y

Complementariedad apoyan la productividad y competitividad de estas empresas, entonces es importante investigar que se puede identificar para que puedan lograr un crecimiento económico sostenible y puedan ser competitivas no solo a nivel nacional sino también en forma internacional.

La importancia de esta investigación radica en que las pymes son empresas de gran relevancia para la economía de los países, para Honduras que actualmente está pasando por una situación económica alarmante, no es la excepción, sobre todo cuando estas empresas son proveedoras de empleo autoempleo, son diversos los factores que las llevan a desaparecer, los cuales serán mencionados y abordados en la presente investigación para conocer los antecedentes, pero más allá como la Interdependencia y Complementariedad apoya la productividad y competitividad de estas empresas, para que puedan lograr un crecimiento económico sostenible y puedan ser competitivas no solo a nivel nacional sino también internacional. 


\section{INTERDEPENDENCIA Y COMPLEMENTARIEDAD ENTRE PYME 'S COMO FACTOR DE LA PRODUCTIVIDAD Y COMPETITIVIDAD}

Revista Economía y Administración, Vol. 9, No. 22018

proviene de una competencia cada vez más

Kerkhoff (2000) encontró que las pymes constituyen entre el 90 y 98 por ciento de las unidades productivas en América Latina, generan alrededor del 63 por ciento del empleo y participan con el 35 y hasta 40 por ciento del Producto total de la región, según coinciden los más recientes estudios de organismos como el Banco Mundial, el Banco Interamericano de Desarrollo y la Comisión Económica para América Latina.

Para que una empresa pueda lograr el éxito requiere de una Interdependencia y Complementariedad optima que le permita ser productivo y competitivo, cabe mencionar que la empresa no es un factor aislado ya que funciona dentro de un sistema al que debe conocer comprender y atender mismo que se compone de clientes, distribuidores, proveedores, competencia y una sociedad que demanda responsabilidad.

Las empresas industriales de los países desarrollados y de los países en vías de desarrollo se ven hoy ante la necesidad imperiosa de incrementar su competitividad. Semejante desafío dura, una "carrera" por adoptar y adaptar modelos de producción "japoneses" y un cambio tecnológico acelerado. $\mathrm{Y}$ en esa carrera están enfrascadas todas las empresas, ya que hasta las posiciones hegemónicas se tornan cada vez más vulnerables.

Los esfuerzos más importantes para elevar la competitividad deben efectuarse a nivel de empresa. Ahora bien, la competitividad internacional no se explica exclusivamente a nivel empresarial. Las empresas se hacen competitivas al cumplirse dos requisitos fundamentales: primero, estar sometidas a una presión de competencia que las obligue a desplegar esfuerzos sostenidos por mejorar sus productos y su eficiencia productiva; segundo, estar insertas en redes articuladas dentro de las cuales los esfuerzos de cada empresa se vean apoyados por toda una serie de externalidades, servicios e instituciones. Ambos requisitos están condicionados a su vez por factores situados en el nivel macro (contexto macroeconómico y políticoadministrativo) y en el nivel meso. (Esser, Competitividad Sistémica: Nuevo Desafío a las Empresas y a la Política, 1996) 


\section{INTERDEPENDENCIA Y COMPLEMENTARIEDAD ENTRE PYME 'S COMO FACTOR DE LA PRODUCTIVIDAD Y COMPETITIVIDAD}

Revista Economía y Administración, Vol. 9, No. 22018

solamente 3,3 puntos, siendo el mercado interno el indicador más bajo del índice. La ineficiencia de la burocracia Situación Competitiva de gubernamental fue votada por los

\section{Centroamérica}

"Tres países de la región subieron de posición en el ranking global de 20162017. Panamá, el país más competitivo de Centroamérica, subió de la posición 50 a la 42 y obtuvo 4,5 puntos de un máximo de 7.

La categoría más débil de Panamá es el tamaño del mercado, en el que anotó 3,5 puntos, donde el tamaño del mercado interno siendo su área particular de debilidad. Además, los ejecutivos encuestados indicaron que la corrupción era el mayor obstáculo para hacer negocios en Panamá.

El segundo país más competitivo de la región es Costa Rica, registrando 4,4 puntos, y bajando dos puestos a la posición número 54. Con una nota de 6,2, el país destaca por su fortaleza en salud y educación, con altos índices de esperanza de vida y matrícula escolar primaria. Al igual que Panamá, la debilidad de Costa Rica es su tamaño de mercado anotando ejecutivos encuestados como el mayor problema para hacer negocios en Costa Rica.

Guatemala ocupa el tercer lugar en la región con 4,1 puntos, manteniéndose en la posición 78 en el ranking global. La salud y educación primaria es el pilar más fuerte del país, logrando un puntaje de 5. El informe destaca la debilidad de Guatemala en cuanto a innovación, indicador en el cual obtuvo su nota más baja de 3,2. Dentro de éste, la principal área de debilidad es la adquisición de productos tecnológicos avanzados por parte del gobierno, ubicándose en el puesto 127 en el mundo para este indicador. El crimen y el robo fueron considerados como el mayor obstáculo para hacer negocios en el país.

\section{Honduras}

Honduras se mantiene en el puesto 88 en el ranking global con 4 puntos, y en cuarto lugar en la región. Al igual que Costa Rica y Guatemala, el pilar con la calificación 


\section{INTERDEPENDENCIA Y COMPLEMENTARIEDAD ENTRE PYME 'S COMO FACTOR DE LA PRODUCTIVIDAD Y COMPETITIVIDAD}

Revista Economía y Administración, Vol. 9, No. 22018

más alta es salud y educación primaria, con 5,6 puntos. Presenta una tasa de

matrícula a nivel escolar primario de $94 \%$, valor que la ubica en la posición 79 a nivel mundial.

El informe refleja tres pilares como los más débiles de Honduras, todos con 3,1 puntos. El primero es el tamaño del mercado debido a su bajo índice de mercado interno. La innovación es el segundo pilar, con una baja calidad de instituciones de investigación científica y poca capacidad de innovación. Por último, el país muestra deficiencias en la preparación tecnológica principalmente debido a los bajos índices de acceso a servicios de internet. Adicionalmente, la ineficiencia de la burocracia del gobierno resultó ser el factor más problemático para hacer negocios en Honduras según ejecutivos encuestados." (Rogers, 2017)

Honduras vive una transición demográfica, debido a que la mayoría de su población es joven $\mathrm{y}$, por tanto, se encuentra en la edad plena para la productividad y el desarrollo de sus capacidades y potencialidades. Según datos del INE, en 2012 un 58\% de la población total tenía menos de 25 años. (Secretaría Técnica de Planificación y Cooperación Externa, 2010).

\section{DEFINICIÓN} DEL

\section{PROBLEMA}

Las Pymes son empresas de gran relevancia para la economía de los países. Y para Honduras no es la excepción, según datos actualizados al 2006 contribuyen con el 38\% del PIB del país (para el 2006 se contaba con 83.347 empresas Pymes formales existentes según datos del INE (INE 2008).

Si se comparan los datos del INE AL 2008 con lo que expone (Maradiaga, 2014) donde indica que las pymes para ese año “aportan el 47\% del Producto Interno Bruto (PIB) del país y emplean el $72 \%$ de la población económicamente activa", se puede observar que el aporte que hacen las PYMEs al país va en incremento.

(Kerkhoff, 2000) encontró que las Pymes constituyen entre el $90-98 \%$ de las unidades productivas en América Latina, generan alrededor del $63 \%$ del empleo y participan con el $35-40 \%$ del Producto 


\section{INTERDEPENDENCIA Y COMPLEMENTARIEDAD ENTRE PYME 'S COMO FACTOR DE LA PRODUCTIVIDAD Y COMPETITIVIDAD}

Revista Economía y Administración, Vol. 9, No. 22018

total de la región (según coinciden los más

recientes estudios de organismos como el Banco Mundial, el Banco Interamericano de Desarrollo y la Comisión Económica para América Latina).

Estrategia y metodología seguir La investigación tiene un enfoque cuantitativo Tipo y Alcance de Investigación Correlacional ya que se pretende relacionar variables y contrastar los resultados para ver cuál es la relación que existe entre las variables. Con un diseño de la Investigación No Experimental Transversal

En cuanto al aporte de Aporte la investigación es constructivista porque pretende aportar conocimiento útil para la Academia, las Empresas y el País utilizando el método dialectico hermenéutico para la comprensión, la explicación y la interpretación de las variables planteadas.

\section{CATEGORÍAS DE ESTUDIO Y VARIABLES}

La presente investigación tiene una categoría empresarial - Económica, relacionada con la sociedad donde el gobierno juega un papel relevante debido a la importancia que representan las pymes que afectan o benefician de manera directa la economía del país.

\section{Propósitos}

A pesar de la importancia que tienen las pymes en Honduras, no se cuenta con información clara sobre la situación de interdependencia, complementariedad, productividad y competitividad que presentan las mismas, para lo cual se realizara en presente estudio que creara las bases para futuras investigaciones y más allá de eso se presentaran sugerencias que apoyen la competitividad de estas unidades y del país en general, evitando de esta forma la alta tasa de mortalidad, que al final influye y crea otros fenómenos negativos en el país, como lo es el aumento de desempleo, bajo nivel productivo, y se ve afectada la situación económica en general del país.

\section{A Nivel de Honduras}

Según el estudio Estado y Competitividad de la Micro, Pequeña y mediana empresa realizado por el (Programa de las Naciones Unidas para el Desarrollo [PNUD], 2003) elaborado por Irene Barquero, las PYMES 


\section{INTERDEPENDENCIA Y COMPLEMENTARIEDAD ENTRE PYME 'S COMO FACTOR DE LA PRODUCTIVIDAD Y COMPETITIVIDAD}

Revista Economía y Administración, Vol. 9, No. 22018

en Honduras

aproximadamente

260,000

establecimientos, que proveen empleo al 45\% de la Población Económica del país. Entre 1996 y 2000, el número de PYMES se vio incrementado en $32 \%$, lo que representa un incremento del empleo generado por el sector de $34 \%$. Aunque no existen datos exactos de la contribución de las PYMES al ingreso doméstico bruto, se estima que, en el ámbito de la región centroamericana, las PYMES contribuyen con aproximadamente el $12 \%$ del total de ingresos.

Parte de la problemática enfrentada al momento de generar estrategias de apoyo al desarrollo de las PYMES en Honduras es la falta de información estadística precisa. Por los momentos la mayoría de instituciones privadas de apoyo al sector basan sus esfuerzos en dos estudios elaborados por una firma de consultoría privada, los que constituyen la mejor base de datos disponible, aunque desgraciadamente solo cubre los sectores del micro y pequeña empresa. Debido a esta falta de información, los datos de dichos estudios serán utilizados como base para el presente estudio.
Con respecto a la participación de las micro y pequeñas empresas (MYPE) en

\begin{tabular}{|c|c|c|c|}
\hline \multicolumn{4}{|c|}{$\begin{array}{c}\text { Empleo En Las PYMES y Porcentaje de Participación } \\
\text { Por Rama de Actividad. Año } 2000 .\end{array}$} \\
\hline $\begin{array}{l}\text { Rama de } \\
\text { Actividad }\end{array}$ & $\begin{array}{l}\text { Número de } \\
\text { empleos pequeña } \\
\text { Empresa }\end{array}$ & $\begin{array}{l}\text { Porcentaj } \\
\text { e de } \\
\text { Participac } \\
\text { ión }\end{array}$ & $\begin{array}{l}\text { Empleo en } \\
\text { la Mediana } \\
\text { Empresa }\end{array}$ \\
\hline Comercio & 35,901 & 31 & \multirow{4}{*}{32,170} \\
\hline Servicios & 19,688 & 17 & \\
\hline Industria & 32,427 & 27 & \\
\hline Otros & 27,795 & 24 & \\
\hline $\begin{array}{l}\text { Total, } \\
\text { empleo } \\
\end{array}$ & 115,811 & & 147,981 \\
\hline \multicolumn{4}{|c|}{$\begin{array}{l}\text { La pequeña y Mediana Empresas en Honduras, } \\
\text { Revista Centroamericana de Economía, Pág. } 80\end{array}$} \\
\hline \multicolumn{4}{|c|}{ érminos de empleo, el total de los dueños } \\
\hline \multicolumn{4}{|c|}{ trabajadores de este segmento como } \\
\hline \multicolumn{4}{|c|}{ porción de la PEA alcanza alrededor del } \\
\hline
\end{tabular}

\section{RELEVANCIA, APORTES DEL INVESTIGADOR Y SUS HALLAZGOS}

Esta investigación es de gran relevancia para los empresarios de las pequeñas y medianas empresas, así como de aquellos empresarios interesados en ser más competitivos a través de mejorar su productividad. Para la academia es de gran relevancia ya que sirve como aprendizaje y un modelo para futuros investigadores.

La investigación es constructivista porque pretende aportar conocimiento útil para la Academia, las Empresas y al País. Se usará un método dialectico hermenéutico para la 


\title{
INTERDEPENDENCIA Y COMPLEMENTARIEDAD ENTRE PYME 'S COMO FACTOR DE LA PRODUCTIVIDAD Y COMPETITIVIDAD
}

\begin{abstract}
Revista Economía y Administración, Vol. 9, No. 22018
comprensión, la explicación y la estudio realizado a empresas interpretación de las variables planteadas, panificadoras. Zuniga, 2013 para lo cual también se contará con Para elevar la competitividad de las entrevistas a expertos que conocen sobre el empresas, se puede lograr a través de tema y que pertenecen a universidades de varias estrategias, La cooperación inter países desarrollados, que puedan explicar empresarial, la complementariedad o la a qué se debe el crecimiento de las interconexión es una importante estrategia empresas Pymes en estos países y cuáles para poder elevar la competitividad de las son las limitantes según su criterio para las empresas e industrias lo cual impacta de empresas Latinoamericanas, y de esta manera contar con un modelo apegado al
\end{abstract} contexto.

También se proporcionará un prototipo de Interdependencia y Complementariedad optima que apoye a las empresas objeto de estudio para ser competitivas, partiendo de investigaciones realizadas por otros expertos, pero de acuerdo con el contexto.

\section{Conclusiones o reflexiones preliminares}

Una importante razón por la cual las empresas no son productivas y tienen bajos niveles de productividad se debe a que muchos emprendedores tienen un producto o servicio, pero no tienen una empresa. Por otro lado;

La planificación es deficiente (el principal problema visto no es que la planificación sea mala en sí, es que no suele haber planificación alguna.) esto tomado del

\section{Notas Explicativas del Autor}

Si las empresas juegan un papel relevante para la economía aun siendo que para las PYMEs representa un verdadero reto el implementar estrategias que apoyen la competitividad de las mimas, y que muchas veces desconocen o les da cierta desconfianza implementar para lograr el éxito que requieren, el tema de la Interdependencia y Complementariedad les es un tanto complicado de comprender o aceptar, pero deben de estar conscientes que en la actualidad requieren de este tipo de estrategias Horizontales que les permita ser productivo y competitivo, cabe mencionar que la empresa no es un factor aislado ya que funciona dentro de un 


\section{INTERDEPENDENCIA Y COMPLEMENTARIEDAD ENTRE PYME 'S COMO FACTOR DE LA PRODUCTIVIDAD Y COMPETITIVIDAD}

Revista Economía y Administración, Vol. 9, No. 22018

sistema al que debe conocer comprender y atender, mismo que se compone de clientes, distribuidores, proveedores, competencia y de una sociedad que demanda responsabilidad.

Cabe mencionar que en el presente artículo solo se está exponiendo la primera etapa de la tesis doctoral, aún se está trabajando en el marco teórico para lo cual se realizaran dos publicaciones más sobre el tema, para que se pueda llegar a la conclusión de la investigación realizada en su totalidad haciendo la propuesta correspondiente en base a la misma.

\section{BIBLIOGRAFÍAS REFERENCIAS}

Centro de Comercio Internacional. (2013).

La Ventaja Competitiva Nacional. Forum de Comercio Internacional.

Instituto Nacional de Estadistica. (2008). Pymes: un Pilar de la Economía Nacional. Tegucigalpa

Maradiaga, J. R. (2014). Las MIPYMES en Honduras. Diario La Tribuna.
Zevallos, E. (2003). Micro, pequeñas y medianas empresas en América Latina. REVISTA DE LA CEPAL 79, 58, 59.

Zuniga, Doris (2013). Estados Financiero como Herramientas para la Toma de Decisiones "Caso Panificadoras de la Ciudad de Tegucigalpa, Honduras". Barquero, Irene (2003), El estado y competitividad de la micro, pequeña y Mediana empresa (1a. ed.). Tegucigalpa: PNUD.

Kerkhoffn, Frans (2000), Honduras: Competitividad de las Mipyme, cap. V.

Rogers, Nicholas (2017), Honduras: La Competitividad de Centroamérica. Informe Global de Competitividad anual. http://americaeconomia-

ca.com/2017/08/18/la-competitividad-decentroamerica/

Klaus Esser / Wolfgang Hillebrand / Dirk Messner / Jörg Meyer-Stamer (1996), Santiago: Competitividad sistémica: Nuevo desafío a las empresas y a la política. Revista de la CEPAL 
INTERDEPENDENCIA Y COMPLEMENTARIEDAD ENTRE PYME 'S COMO

FACTOR DE LA PRODUCTIVIDAD Y COMPETITIVIDAD

Revista Economía y Administración, Vol. 9, No. 22018 
INTERDEPENDENCIA Y COMPLEMENTARIEDAD ENTRE PYME 'S COMO

FACTOR DE LA PRODUCTIVIDAD Y COMPETITIVIDAD

Revista Economía y Administración, Vol. 9, No. 22018 